\title{
INTRODUCTION OF THE PHONE-CONCEPT INTO POLE FIGURE INVERSION USING THE ITERATIVE SERIES EXPANSION METHOD
}

\author{
M. DAHMS \\ GKSS-Forschungszentrum, Max-Planck-Straße, D-2054 Geesthacht
}

(Received October 31, 1991)

\begin{abstract}
The phone-concept as it is used in the various kinds of probabilistic methods can easily be applied to the iterative series expansion method for quantitative texture analysis. Only slight modifications of the existing routines are necessary. The advantages of this concept are demonstrated by a mathematical and an experimental example.
\end{abstract}

KEY WORDS Positivity method, iterative series expansion method, phone, isotropic texture component

\section{INTRODUCTION}

It is the major drawback of the series expansion method that after pole figure inversion, negative pole densities may appear in recalculated pole figures. In the case of incomplete pole figures, this may happen in the unmeasured range of the measured pole figures (Dahms, 1987). If only a small number of pole figures is measured, strong negative intensities may occur in recalculated unmeasured pole figures (Dahms \& Bunge, 1988a). Also the iterative series expansion method, the so-called "positivity method" (Dahms \& Bunge, 1989), does not necessarily result in completely positive recalculated pole figures (Dahms, 1992). If there are negative pole densities in recalculated pole figure, being physically impossible, it is impossible to obtain a totally positive complete ODF. It is the aim of this paper to show that a slight modification of the positivity method leads to a considerable improvement of the pole figure inversion with respect to positivity of recalculated pole figures.

\section{GENERAL CONSIDERATIONS}

In the positivity method (Dahms \& Bunge, 1989), after a first approximation of the series expansion coefficients $C_{l}^{\mu \nu}$, the unmeasured range of the measured pole figures and additional unmeasured pole figures are calculated. Any of these pole densities below zero is set to zero, and using these corrected pole figures, a second approximation of the $C_{l}^{\mu \nu}$ is calculated. Using this loop, an iterative procedure is defined, which is the "even" part of the positivity method. The iteration is stopped, if no further negative values occur, no further improvement of the $C_{l}^{\mu v}$ is obtained or if a time barrier is reached. 
In the case of textures with an isotropic component $r$ or "phone" (Matthies, 1984), this procedure will obviously not lead to a correct pole figure inversion if just a few pole figures are measured. In the best case, unmeasured recalculated pole figures will contain regions with zero pole density if the first approximation of the $C_{l}^{\mu \nu}$ leads to negative pole densities.

The situation is improved if not only negative pole densities are set to zero but densities less than $r$ are set to $r$. In general, if an infinite number of additional pole figures is introduced, i.e., the whole inverse pole figure is covered, the original texture will be exactly reproduced. In fact, the boundary condition "no pole density less than $r$ " is a higher constraint than the positivity condition.

In practice, it is not known whether there is a phone present or not. Also, if the "even" ODF has an isotropic component, i.e., the absolute minimum of all pole figures is greater than zero, just the range of ambiguity of the complete ODF is touched (Matthies, 1979). In case of a limited number of measured pole figures, the problem is even worse. The minimum of all pole figures is a value between zero and the minimum pole density of the measured pole figures.

In the present variant of the iterative series expansion method, the value $r$ is entered manually in the routine and chosen according to the experience of the operator. As a criterion for a proper choice of $r$, the "simplicity" of the recalculated pole figures is chosen, i.e., a texture should be interpreted by a small number of texture components. Here, some relation to the component method (Helming and Eschner, 1990) can be seen.

\section{EXAMPLES}

In order to show the versality of the suggested generalization of the positivity method, two examples will be presented. The first one is a synthetic (001)[100]texture with a gaussian spread of $12.5^{\circ}$ and an isotropic component of $r=0.5$ assuming orthorhombic crystal and sample symmetries, see Dahms and Bunge (1988b). The second one is that of natural quartz as published by Braun et al. (1991).

\section{Synthetic texture}

As an extreme case, only the three (100), (010) and (001) pole figures were given. For a degree of series expansion of $L=22$, at least 12 pole figures are necessary. For pole figure inversion, the (111), $3\{110\}, 6\{210\}, 3\{311\}$, and $6\{321\}$ pole figures were introduced in the calculation as described by Dahms and Bunge (1989). Hence, 19 additional pole figures were checked for positivity during the iterative procedure. The first calculation was done with only positivity assumed, the second calculation incorporated the isotropic component $r=0.5$.

After pole figure inversion, the general axis distribution function (Bunge, 1982) was calculated in steps of $5^{\circ}$ for all crystal and sample directions, i.e., for each crystal direction, a complete pole figure was calculated. In Figure 1, the minimum value of each individual pole figure is represented in inverse pole figures. Figure 1a shows the result for simple positivity $r=0$ and, Figure 1a shows the result for $r=0.5$. It can be seen that for $r=0$, considerable negative intensities are present 
a)

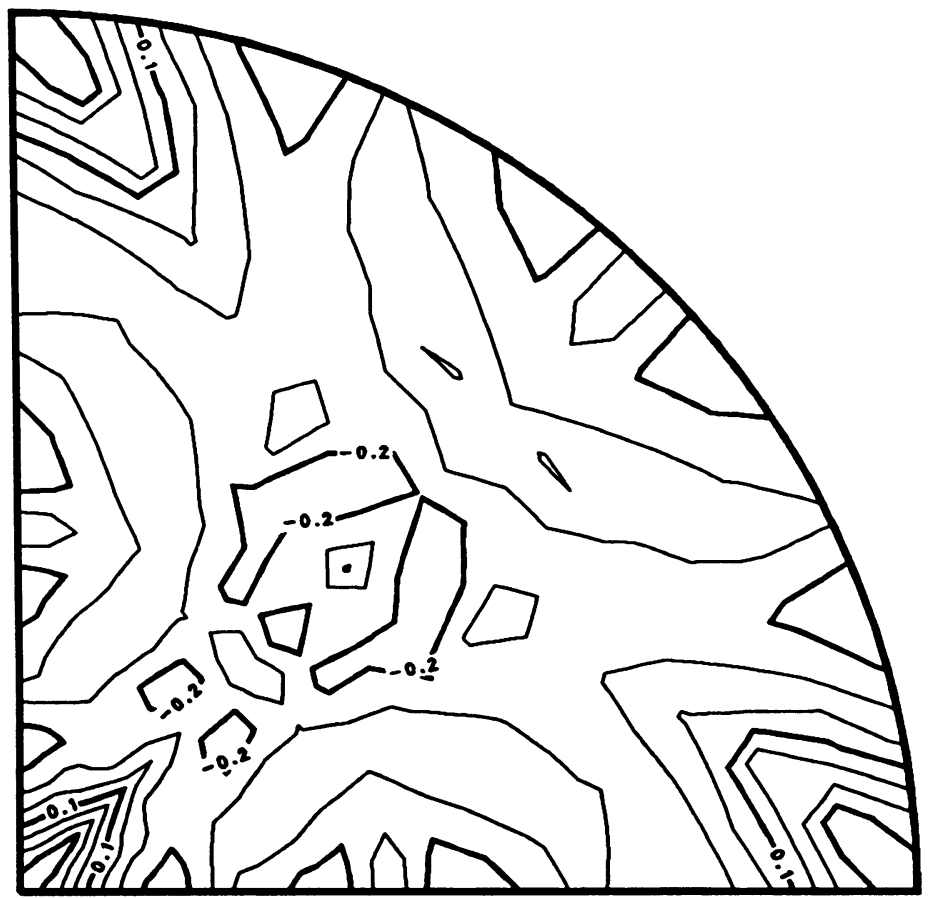

ग)

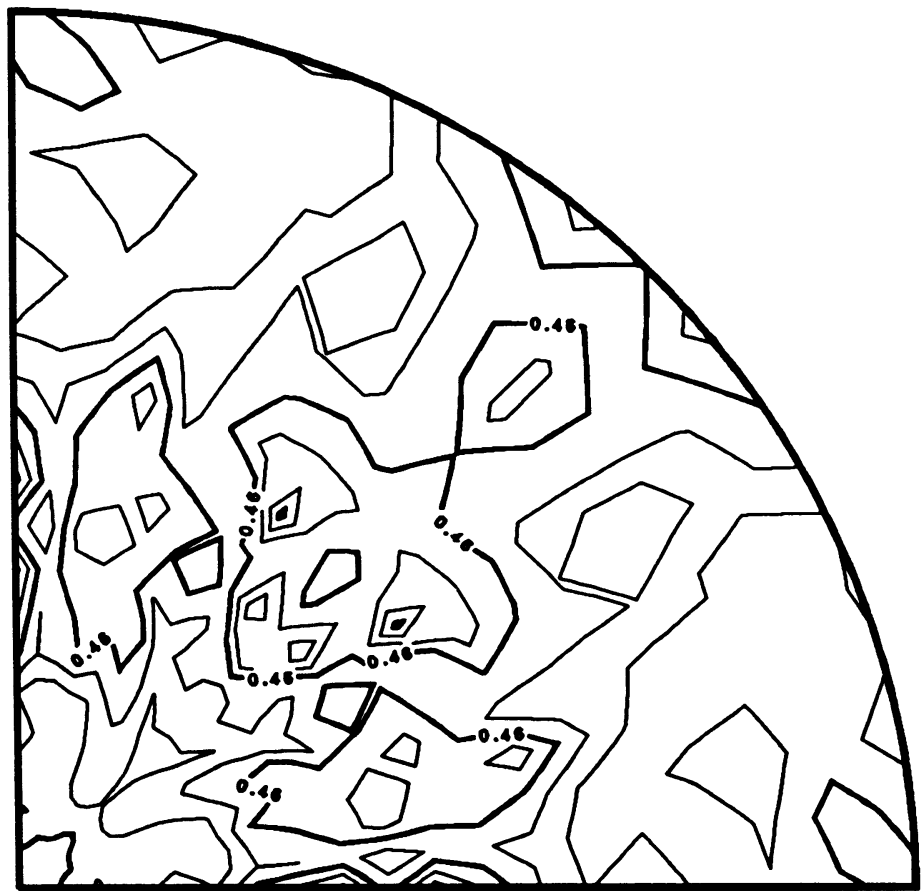

PMAX $=0.50$

PMIN $=-0.28$ CONTOUR LEVELS -0.2 ,

-0.1 ,

0.0 ,

0.1 ,

0.2 ,

0.3 ,

$0.4 X$ RANDOM
PMAX $=0.50$

PMIN $=0.43$

CONTOUR LEVELS

0.43 ,

0.44 ,

0.45 ,

0.46 ,

0.47 ,

0.48 ,

$0.49 \times$ RANDOM

Figure 1 Minimum values of all recalculated pole figures represented in an inverse pole figure for a synthetic (001)[100]-texture with a gaussian spread of $12.5^{\circ}$ and an isotropic component of $r=0.5$ assuming orthohombic crystal and sample symmetries after pole figure inversion of the three (100), (010) and (001) pole figures. (a) Positivity method. (b) Positivity method including phone of 0.5 . 


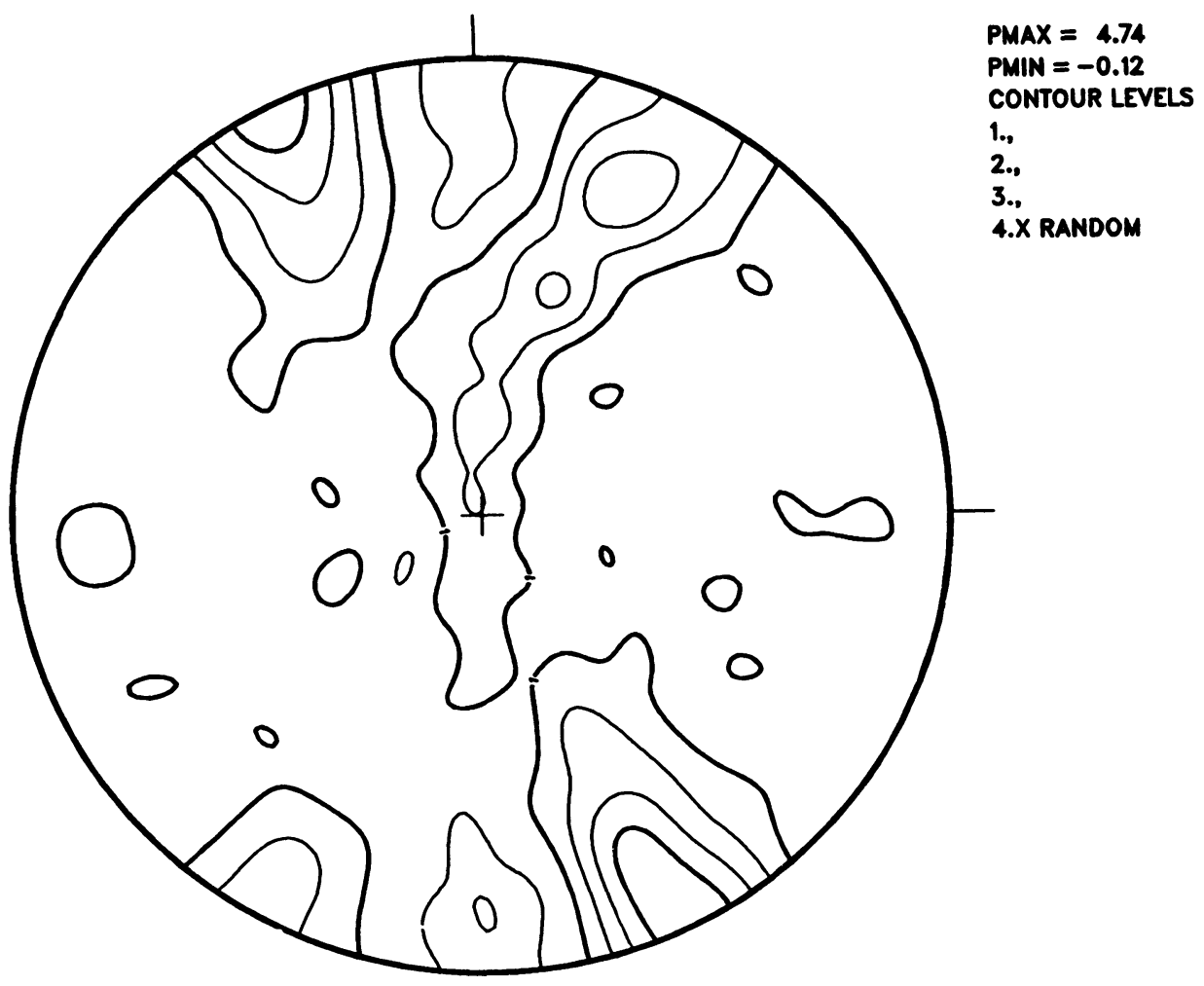

Figure 2a Calculated (001) pole figure of a natural quartz specimen. Positivity method.

in pole figure which were not incorporated in the iterative procedure. For $r=0.5$, the minimum value of all recalculated pole figures is 0.43 . The remaining error of 0.07 is due to truncation of the iteration. It is an order of magnitude smaller than the error of 0.75 occurring in the simple positivity method.

\section{Real texture}

Using seven measured and 16 additional pole figures, pole figure inversion was carried out up to $L=22$. The (001) pole figure cannot be measured using $X$-rays, but was one of the additional pole figures. For geologists, it carries the major texture information.

In Figure 2 the calculated pole figures for $r=0$ (Figure 2a) and $r=0.5$ (Figure $2 b)$ are shown. There is no serious difference, except the minimum values which were raised from -0.12 to 0.21 . Additionally, most of the smaller peaks taking place for $r=0$ disappear in the case of $r=0.5$. Obviously, the introduction of a positive minimum value acts as a tension towards real positivity and smoothness. It is clear from these results that an over-estimation of $r$ does not lead to minimum values in pole figures very close to $r$. 


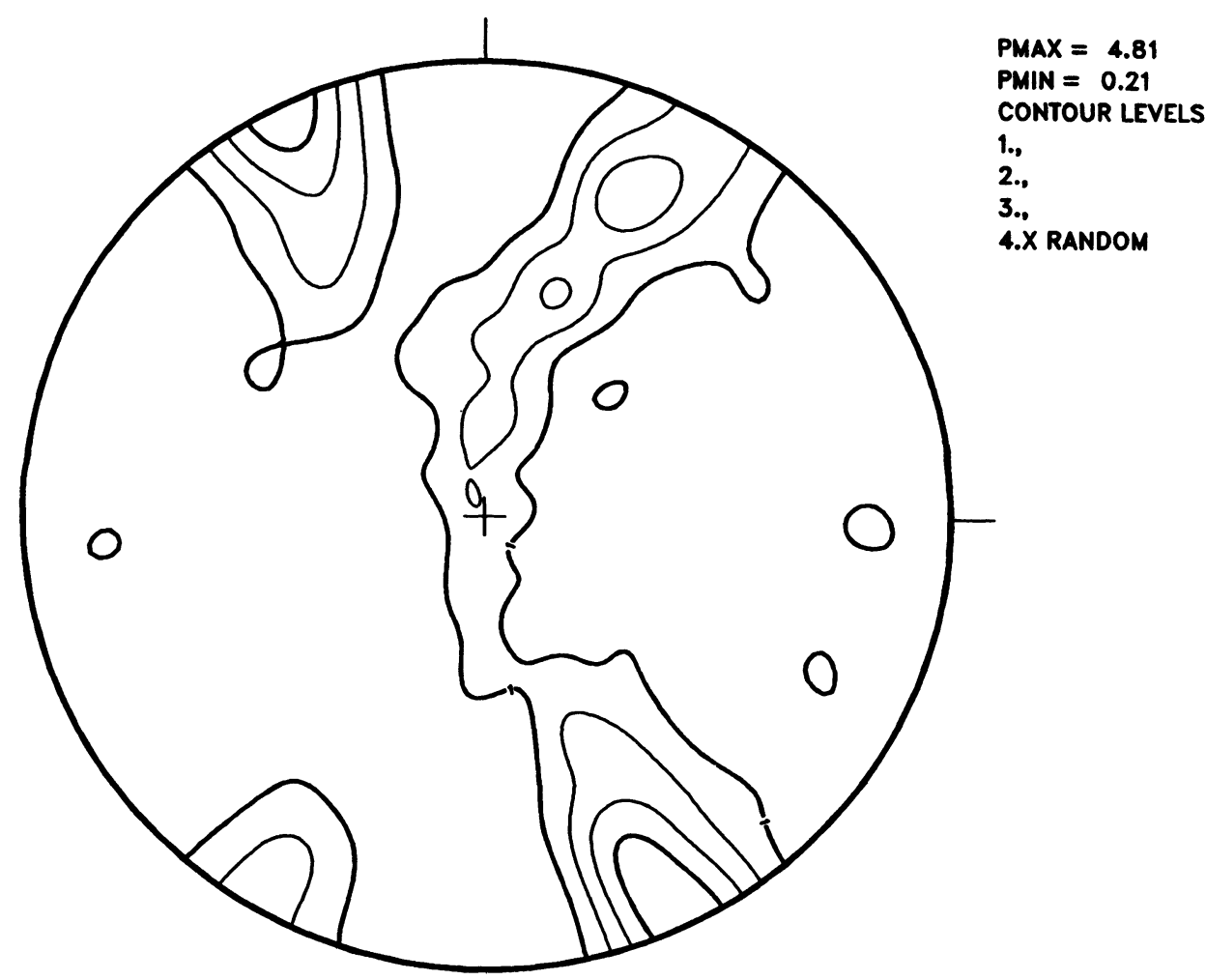

Figure 2b Calculated (001) pole figure of a natural quartz specimen. Positivity method including phone of 0.5 .

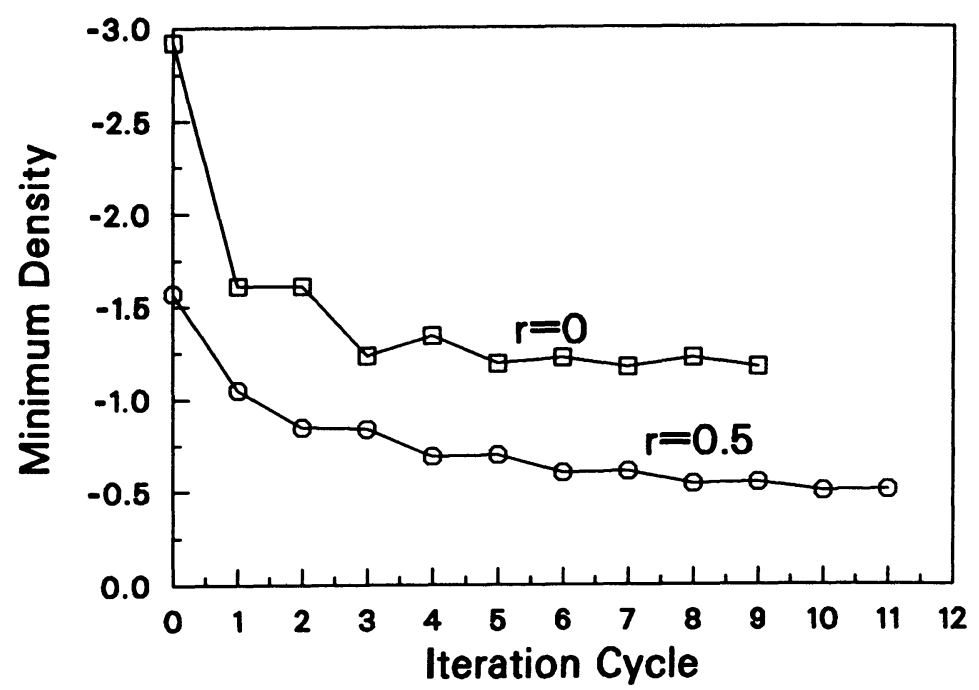

Figure 3 Development of the minimum value of the ODF during the iterative procedure for the determination of odd-order coefficients in the case of the natural quartz texture starting with an "even" ODF including completely positive pole figures $(r=0.5)$ or including pole figures with negative regions $(r=0)$. 
If odd-order coefficients are determined using the positivity method (Dahms \& Bunge, 1989), the minimum value of the complete ODF remains less than -1 for the case of $r=0$, i.e., the negative values in the pole figures are transferred to the ODF. For $r=0.5$, a minimum value of -0.5 is reached after 10 iterative cycles, see Figure 3. By separate calculations (Dahms, 1991), it has been shown that this minimum is due to series truncation effects. The difference between minimum values of about 0.5 is in the range of the assumed isotropic texture component $r$, and it is also in the range of the raised minimum of the (001) pole figure.

Here, we have a typical example, how a slight improvement of the "even" ODF leads to a considerable improvement of the complete ODF as it has been shown by Wagner et al. (1990).

\section{CONCLUSIONS}

The present introduction of Matthies' phone-concept into the series expansion method has two advantages:

-From a theoretical point of view, proper pole figure inversion of pole figures with a "true" isotropic texture is possible, even if just a limited number of pole figures is available.

-From a practical point of view, all recalculated pole figures can be made positive by the constraint of an artificial isotropic component. This leads to a better determination of the odd-order coefficients using the positivity method.

\section{References}

Braun, G., Siegesmund, S. and Dahms, M. (1991). The influence of quartz textures on the seismic anisotropy in lower crystal granulites. Journal of Structural Geology 13, 955-966.

Dahms, M. (1987). Spezielle mathematische Methoden der Texturanalyse und ihre Anwendungen unter besonderer Berücksichtigung der intermetallischen Phasen, Dr.-Ing. Thesis, TU Clausthal.

Dahms, M. (1991). unpublished results.

Dahms, M. (1992). The iterative series expansion method for quantitative texture analysis, part II: applications. J. Appl. Cryst. 25, accepted for publication.

Dahms, M. and Bunge, H. J. (1988a). ODF calculation by series expansion from incompletely measured pole figures using the positivity condition, part II-all crystal symmetries. Textures and Microstructures 8 \& 9, 97-114.

Dahms, M. and Bunge, H. J. (1988b). A positivity method for the determination of complete orientation distribution functions. Textures and Microstructures 10, 21-35.

Dahms, M. and Bunge, H. J. (1989). The iterative series expansion method for quantitative texture analysis-part I: general outline. J. Appl. Cryst. 22, 439-447.

Helming, K. and Eschner, T. (1990). A new approach to texture analysis of multiphase materials using a texture component model. Crys. Res. Technol. 25, K203-K208.

Matthies, S. (1979). On the reproducibility of the orientation distribution function of texture samples from pole figures (ghost phenomena). Phys. Stat. Sol. B 92, K135-138.

Matthies, S. (1984). The present situation in the field of ODF reproduction and ghost correction. In ICOTOM 7, Proc. 7th Intern. Conf. on Textures of Materials, edited by C. M. Brakman, P. Jongenburger and E. J. Mittenseijer, pp. 737-751, Netherlands Society for Materials Science: Noordwijkerhout.

Wagner, F., Humbert, M., Esling, C. \& Muller, J. (1990). Determination of complete ODFs under assumption of a minimal value. In ICOTOM 9, Proc. Mih Intern. Conf. on Textures of Materials Avignon, France, in the press. 